\title{
Artificial Intelligence (AI) Techniques Applied for the Development of a Clinical Decision Support System (CDSS) for Diagnosing Ischemic Heart Disease (IHD)
}

\author{
K.Rajeswari \\ Associate Professor, \\ PCCOE,Pune University, \\ Pune, India
}

\author{
V.Vaithiyanathan \\ Associate Dean, \\ Research School of \\ Computing, \\ Sastra University,India
}

\author{
Deepa Abin \\ Assistant Professor, \\ PCCOE,Pune University, \\ Pune, India
}

\begin{abstract}
Ischemic Heart Disease (IHD) is difficult to diagnose since most of the symptoms and clinical presentations are similar to other diseases. It is a very common, harmful disease, which is identified mostly during the mortality of an individual. The objective is to build a clinical decision support system, which will diagnose the presence of IHD with an integrated automated classifier using Artificial Intelligence (AI) techniques. A retrospective data set that included 800 clinical cases was taken for the work. A total of 88 sets were discarded during pre-processing. Tests were run on 712 cases using the Weka classifiers available in Weka 3.7.0. Out of 113 classifiers, 16 were identified to be the best based on the following parameters: sensitivity, specificity, accuracy, Fmeasure, kappa statistic, correctly classified cases, time taken to run the model, and the Receiver Operating Characteristic (ROC) curve. The diagnoses made by the Clinical Decision Support System (CDSS) were compared with those made by physicians during patient consultations. The KSTAR algorithm showed the best diagnoses with the highest accuracy $97.32 \%$, sensitivity $98 \%$, specificity $97 \%$ kappa 0.95 , and ROC 0.995. The authors thus conclude that a CDSS can be developed to assist expert physicians in separating the positive and the negative cases of heart disease.
\end{abstract}

\section{General Terms}

Artificial Intelligence,Classification

\section{Keywords}

Clinical Decision Support System (CDSS); Artificial Intelligence (AI) techniques; Ischemic Heart Disease (IHD); Sensitivity; Specificity; Accuracy; F-measure; Kappa statistic; Receiver Operating Characteristic (ROC).

\section{INTRODUCTION}

Ischemic Heart Disease (IHD) is difficult to diagnose and very commonly identified only during the mortality of an individual.According to World Health Organisation (WHO) in India $70 \%$ of coronary death occurs in subjects less than 70 years of age but in North America and Western Europe same percentage of death occur in subjects older than 70 years.[1]. In India, Coronary Heart Disease (CHD) is an epidemic [1].Diagnosis criteria in western medicine is refered as "naming and diagnosis criteria of ischemic heart disease" issued by the International Society of Cardiology and the Joint Subject Team on the standardisation of clinical naming in the WHO [3]. Among Indians, CHD has reached the level of epidemic [4]. With increasing number of CHD cases India is facing a major shift in health [5]. The trend released by WHO
Statistics 2012 is a worrying factor for India [6]. Between the years 1960 and 2000 the prevalence of CHD has raised two fold in rural areas and six fold in urban areas among the adults over 20 years of age [7] and compared to general population of United States the pervasiveness of CHD is approximately four times higher in Indians [8]. Compared to the general population in the United States, the prevalence of CHD in Asian Indians is approximately four times higher [8].

According to WHO statistics [7] in India about 24\% of men and $22.6 \%$ of women aged 25 year and above are suffering from high blood pressure (BP). Over one in ten men and women aged 25 years and above have high blood sugar. More women in India ( $2.5 \%$ of adults aged 20 years and above) are obese compared to Indian men $(1.3 \%)$. Almost one in five (19\%) boys, aged 13-15 years (adolescents), and 8\% girls smoke tobacco. Disparities between countries exist because of cardiovascular diseases [9], with respect to Body Mass Index (BMI). These findings support the fact that obesity may be a pointer to eventual heart disease. As compared to other population, Indian population has higher long-term case fatality following acute coronary syndrome [11]. In Indian population the socio-economic gradient is the major CHD risk factor [12][13].

As per the Medical Council of India, the total number of registered allopathic doctors in the country is as little as under 700,000 . Out of 1.22 billion 72 population, $72.2 \%$ lives in villages and remaining $27.8 \%$ lives in towns and urban area [14]. According to this statistic there is only one allopathic doctor for 2000 patients [15]. This work is expected to help in the classification of the risk levels of the heart disease that causes sudden death. Knowing the risk levels, people can take adequate measures to meet the doctor depending on the severity of the disease, or follow strict dietary rules to avoid problems in future.

\section{MATERIALS AND METHODS}

This study, approved by the Madras Medical College and the SASTRA University (Shanmugha Arts, Science, Technology $\&$ Research Academy), was conducted in the Cardio Thoracic Department of the Madras Medical College. The development of a CDSS for diagnosing IHD consisted of four phases: I, II, III, and IV as in Figure 1. In Phase I, the authors developed an attribute set system for IHD. In Phase II, the authors developed, assessed, and implemented a system for acquisition and retrieval of clinical data in the outpatient clinic. Training and testing databases were constructed here. In Phase III, the data is coded. A training database was tested with a set of automated classifiers. In Phase IV, the authors evaluated the developed CDSS. In this paper, the authors have 
designed a heart system enquiry diagnosis scale [16], in which the symptoms have been defined clearly, and the detailed collecting methods have been listed. The total dataset is divided into training and testing data. A 10-fold cross validation has been used. The different classifiers of Weka are trained using the training set and then, the sensitivity and the specificity of the classifier (once trained) are evaluated on the test set. This evaluation is repeated ten times, alternating the test set used each time. This method reinforces the validation process, so that the sensitivity and specificity values are the average of the ten validation folds [17]. A training set is a set of data used to discover potentially predictive relationships between the input and output. The training set is used by the Weka classifiers to build a model. This model is used to predict the output for the test attributes.

\subsection{Phase I - Attribute construction}

The first stage of the work is attribute identification. Various literature and bench-mark UCI datasets [18] have been analysed and discussed with experts. The attributes, which will help in supporting the clinical decision, will be designed as shown in Table 1 . These attributes can be easily collected through medical camps in a particular area. The method is feasible under the assumption that people are already familiar with the presence of cholesterol and diabetes. In future, these measures as values may be obtained through instruments available in the market.

\subsection{Phase II - Database construction}

A retrospective database (DB1) comprising the firstappointment record of 712 clinical cases was created for algorithm training. Confirmed IHD cases accounted for $68 \%$ of all records, while the remaining diagnoses had signs, symptoms and complaints that were shared with clinical cases of IHD (non-IHD group; NIHD). Patients in the IHD group were classified according to their ECG results, based on the gold standard [2]. Similarly, a testing database (DB2) was created for classifier evaluation. An experimental model was designed to determine the classifiers that were to be integrated into CDSS under development. Automated classifiers available from the Waikato Environment for Knowledge Analysis (Weka 3.7.0) software [19] were tested. Weka is a comprehensive set of libraries organised in Java packages of different classes that implements machine learning and data mining algorithms [11]. A total of 16 attributes (nominal, numeric) of signs, symptoms, and high-risk groups were listed (Appendix A). All clinical data relating to medical histories were coded and given ' $y$ ' (yes) or ' $n$ ' (no) values depending on whether the patient had a certain symptom. Certain attributes like height, weight, BMI, systolic and diastolic BP were numeric values. The target variable was the diagnostic variable out, with Risk or No Risk categories.

\subsection{Phase III - Training and testing classifiers}

AI Techniques

1. Lazy Classifiers: All the training samples are stored till a new sample needs to be classified. This way lazy classifiers are varying from decision tree classifiers, which build a general model before receiving new samples. In the Weka IBk, the authors use the Locally Weighted learning (LWL), KSTAR, and Lazy Bayesian Rules (LBR) classifiers. KSTAR has demonstrated better accuracy for our dataset.
2. Artificial Neural Net (ANN): It is like the human natural neural network with a distributed parallel processor made up of neurons, which are small processing units which have the ability to store knowledge from a database. This knowledge is used for classifying a new data. In this study, the parameters of learning rate (L), varying between 0.3 and 0.5 , momentum (M), varying between 0.2 and 0.5 , and 500 epochs to training (default), were applied to the algorithm Multilayer Perceptron (MLP).

3. Decision Trees: A sequence of tests on attributes using "if..then..else" is performed for classification of tuples according to class label, Here, the ADTree [19], SimpleCart [11], and J48 algorithms, which are trees of C4.5 type [12] are used.

4. Bayesian Classifiers: By assuming the conditional independence of the attributes in the NaiveBayes, NaiveBayesSimple, and BayesNet algorithms [16], by calculating the probability for each class, the classification was achieved. The algorithms AODE [14] and AODEsr [17] minimised the presupposition of conditional independence, thereby resulting in better precision.

5. Support Vector Machines (SVMs): Classification here was achieved by separating the data according to the variation in the parameters of a support vector. In this study, different kernels (polykernel, radial basis function, sigmoid and linear) were used with different penalty parameters for the error term (C) and kernel parameter $(\mathrm{G})$. The parameters $\mathrm{C}$ and $\mathrm{G}$ are varied between $1 \times 10-10$ and $1 \times 1010$ [23].

6. K-nearest Neighbours: According to the distance, mostly Euclidean distance, classification according to the closest data point is done. The algorithm LBR [20] is a Bayesian classifier in which, lazy learning techniques are applied to induce Bayesian rules. In this study, in the algorithm IBk [21], the number of neighbours $(\mathrm{k})$ varied between 1 and 20 . In the algorithm KSTAR [19], the parameter for overall blending (B) varied between 10 and 100 . The algorithm (LBR) was tested with the standard parameters.

7. The variability of the algorithms for each technique and the variations in the parameters resulted in 59 models. From these, 16 best models were tabulated in Table 2. In the Data Preprocessing the values were discretised. In Weka 3.7.0, the cross-validation test was undertaken to estimate the accuracy rate of a classifier with the standard parameters and a 10- fold cross-validation for accuracy estimation. It is understood that various techniques showed different accuracies with different datasets. Our dataset had an accuracy of $97.02 \%$.

\section{Algorithm selection}

The algorithm with the greatest area under the ROC curve (AUC) was selected. The ROC curve was a graphical plot of sensitivity versus one minus specificity, and was used to evaluate classification and prediction models [19]. In the Weka 3.7.0 software, the AUC is calculated by means of the sensitivity and specificity of each of the folds used in training the classifier. The CDSS was designed such that it would issue a warning when the probability of IHD occurrence went above $50 \%$.

\subsection{Phase IV - CDSS evaluation}

The CDSS was then evaluated using the testing dataset DB2. The data relating to each clinical case in DB2 were recorded in the CDSS. The probabilities of IHD occurrence were 
recorded for comparison against the diagnostic intuition that had been recorded by a medical provider during a consultation.

Statistical analysis

Cross-validation with ten subsets (ten-fold cross-validation) was used for testing. To evaluate and compare algorithms with regard to the accuracy of the classification, the parameters used for selecting the algorithm were the highest values for the AUC, sensitivity, specificity and accuracy rate [22]. A comparative analysis between the AUC results for each algorithm before and after the variable selection was performed to determine the best parameters for the algorithm to be selected. Kappa statistics were used to evaluate the agreements between the CDSS and the gold standard: between the physician's impressions, i.e. relating to the diagnostic impression recorded by physicians and their tutors during consultations, and the gold standard, and between the CDSS and the physician's impression. In this manner, the diagnostic reliability of the CDSS is assessed.

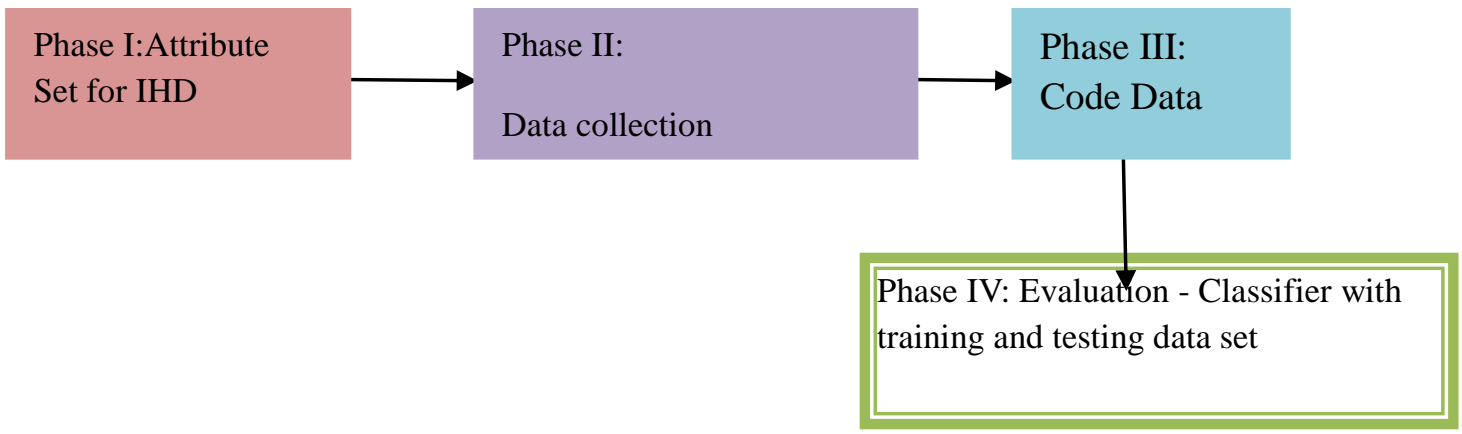

Figure 1. The four phases of CDSS for IHD classification

Table 1. Attributes identified for heart disease identification

\begin{tabular}{|c|c|c|}
\hline No. & Name & Description \\
\hline 1 & Age & Age in years \\
\hline 2 & Sex & $1=$ male $; 0=$ female \\
\hline 3 & Menopause & $0=$ No $; 1=$ yes \\
\hline 4 & Height & $\leq 140 \mathrm{~cm}, 141 \mathrm{~cm}-170 \mathrm{~cm}, \geq 171 \mathrm{~cm}$ \\
\hline 5 & Weight & $\leq 50 \mathrm{Kg}, 51 \mathrm{Kg}-75 \mathrm{Kg}, \geq 76 \mathrm{Kg}$ \\
\hline 6 & BMI & Body Mass Index \\
\hline 7 & Waistcircum & Measure of circumference of waist \\
\hline 8 & SBP & Systolic Blood Pressure \\
\hline 9 & DBP & Diastolic Blood Pressure \\
\hline 10 & Diabetes & Presence is treated as 1 , absence as 0 . \\
\hline 11 & Chol & Presence of cholesterol is treated as 1 , absence as 0 . \\
\hline 12 & Thy & Presence is thyroid is treated as 1 , absence as 0 . \\
\hline 13 & Per_hab & Personal habits of drinking/smoking is recorded as 1,0 otherwise. \\
\hline 14 & Fam_hist & Family history of heart disease presence is treated as 1 else as 0. \\
\hline 15 & Type A & Type A personality is treated as 1,0 otherwise. \\
\hline 16 & Out & $\begin{array}{l}\text { Output, No risk as } 0 \text {, Low risk as } 1 \text {, Medium risk as } 2 \text { and High risk as } 3 \text {.Later for } 2 \text { class } \\
\text { problem, No risk as } 0 \text { and the Low, Medium and High as } 1\end{array}$ \\
\hline
\end{tabular}


Table 2. Best 16 Classifier Models

\begin{tabular}{|ll|}
\hline \multicolumn{2}{|c|}{ Classifier Name } \\
K star & NNge \\
IBK & Decorate \\
IB1 & Classificatior Name \\
RandomTree & FT \\
Id3 & NB \\
RandomCommittee & RotationForest \\
Rules & LMT \\
RandomForest & MLP \\
\end{tabular}

Table 3. Performance measures of the best $\mathbf{1 6}$ Classifier Models

\begin{tabular}{|c|c|c|c|c|c|c|}
\hline S.No. & Classifier Name & $\begin{array}{l}\text { Correctly } \\
\text { classified }\end{array}$ & $\begin{array}{l}\text { Incorrectly } \\
\text { classified }\end{array}$ & Sensitivity & Accuracy & Precision \\
\hline 1 & KSTAR & $97.32 \%$ & $2.68 \%$ & 97.80 & 97.32 & 97.64 \\
\hline 2 & IBK & $97.22 \%$ & $2.78 \%$ & 97.16 & 97.22 & 98.14 \\
\hline 3 & IB 1 & $96.83 \%$ & $3.17 \%$ & 97.30 & 96.83 & 97.30 \\
\hline 4 & RandomTree & 97.123 & 2.877 & 97.15 & 97.12 & 97.97 \\
\hline 5 & Id 3 & $97.22 \%$ & $2.68 \%$ & 97.47 & 97.32 & 97.97 \\
\hline 6 & $\begin{array}{l}\text { RandomCommitte } \\
\text { e }\end{array}$ & $97.22 \%$ & $2.78 \%$ & 97.16 & 97.22 & 98.14 \\
\hline 7 & Rules & $96.92 \%$ & $2.88 \%$ & 95.62 & 97.12 & 99.66 \\
\hline 8 & RandomForest & $97.02 \%$ & $2.98 \%$ & 97.15 & 97.02 & 97.80 \\
\hline 9 & NNge & $97.02 \%$ & $2.98 \%$ & 97.15 & 97.02 & 97.80 \\
\hline 10 & Decorate & $95.83 \%$ & $4.17 \%$ & 96.93 & 95.83 & 95.95 \\
\hline 11 & $\begin{array}{l}\text { ClassificationVia } \\
\text { Regression }\end{array}$ & $95.54 \%$ & $4.46 \%$ & 96.28 & 95.54 & 96.11 \\
\hline 12 & FT & $95.83 \%$ & $4.17 \%$ & 96.93 & 95.83 & 95.95 \\
\hline 13 & NB & $95.83 \%$ & $4.17 \%$ & 96.93 & 95.83 & 95.95 \\
\hline 14 & RotationForest & $95.83 \%$ & $4.17 \%$ & 96.45 & 95.83 & 96.45 \\
\hline 15 & LMT & $96.73 \%$ & $3.27 \%$ & 97.13 & 96.73 & 97.30 \\
\hline 16 & MLP & $97.02 \%$ & $2.98 \%$ & 97.31 & 97.02 & 97.64 \\
\hline
\end{tabular}




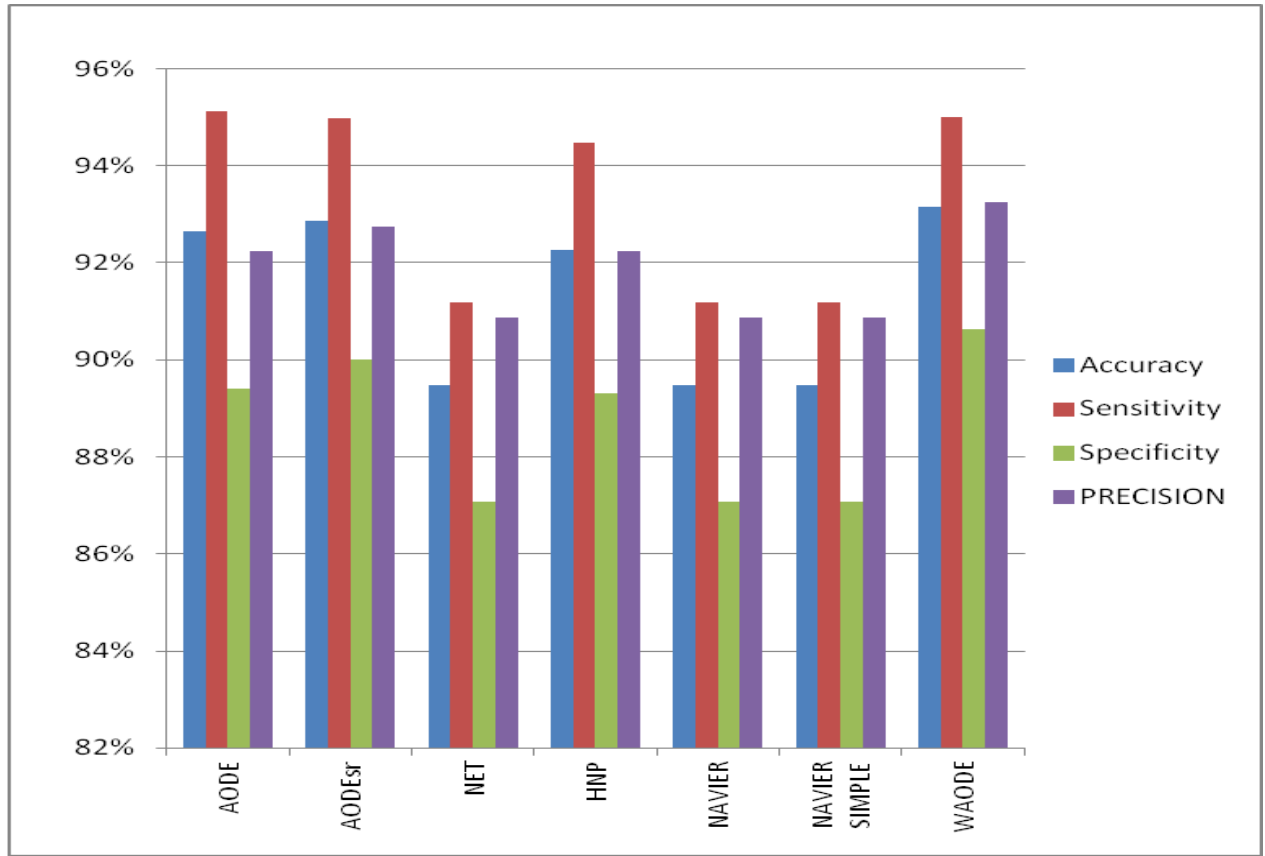

Figure 2. Comparison of Bayes classifiers

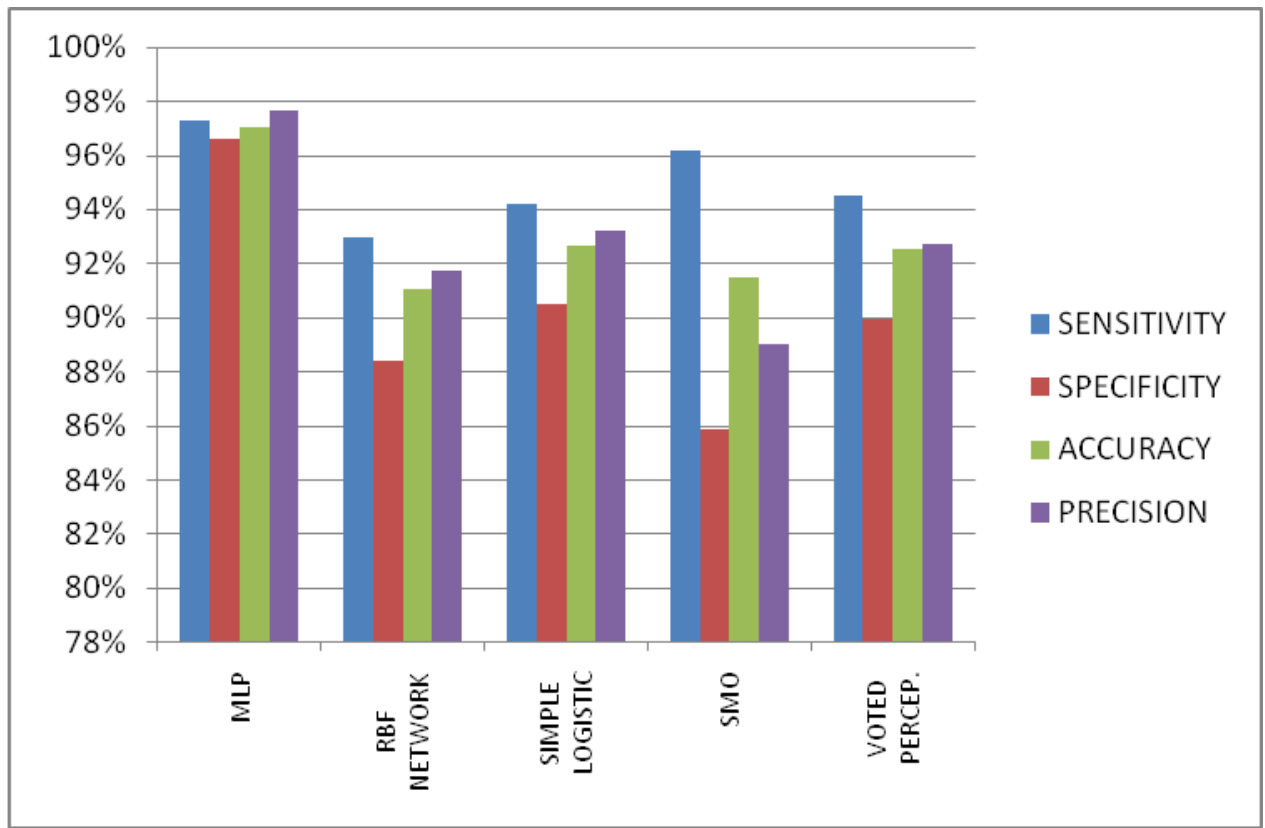

Figure 3. Comparison of Functions classifiers 


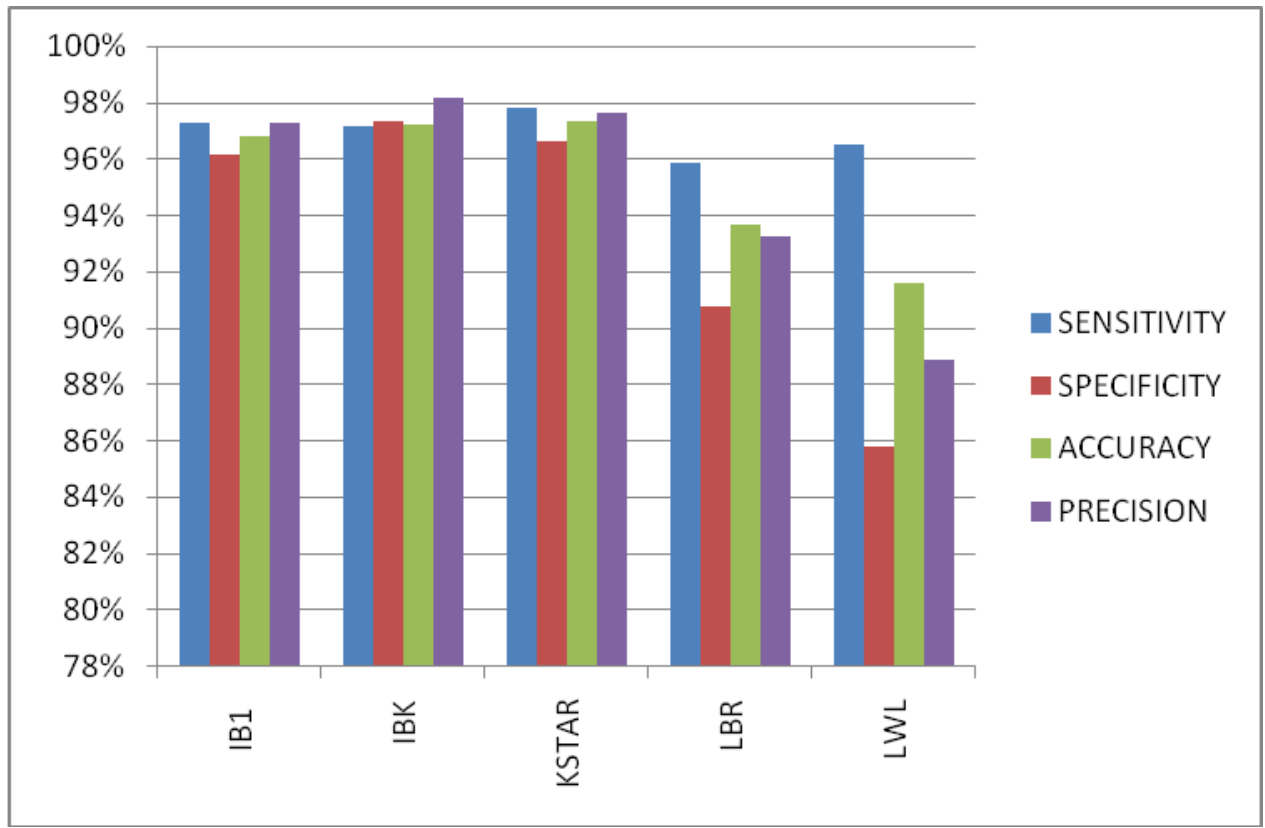

Figure 4. Comparison of Lazy classifiers

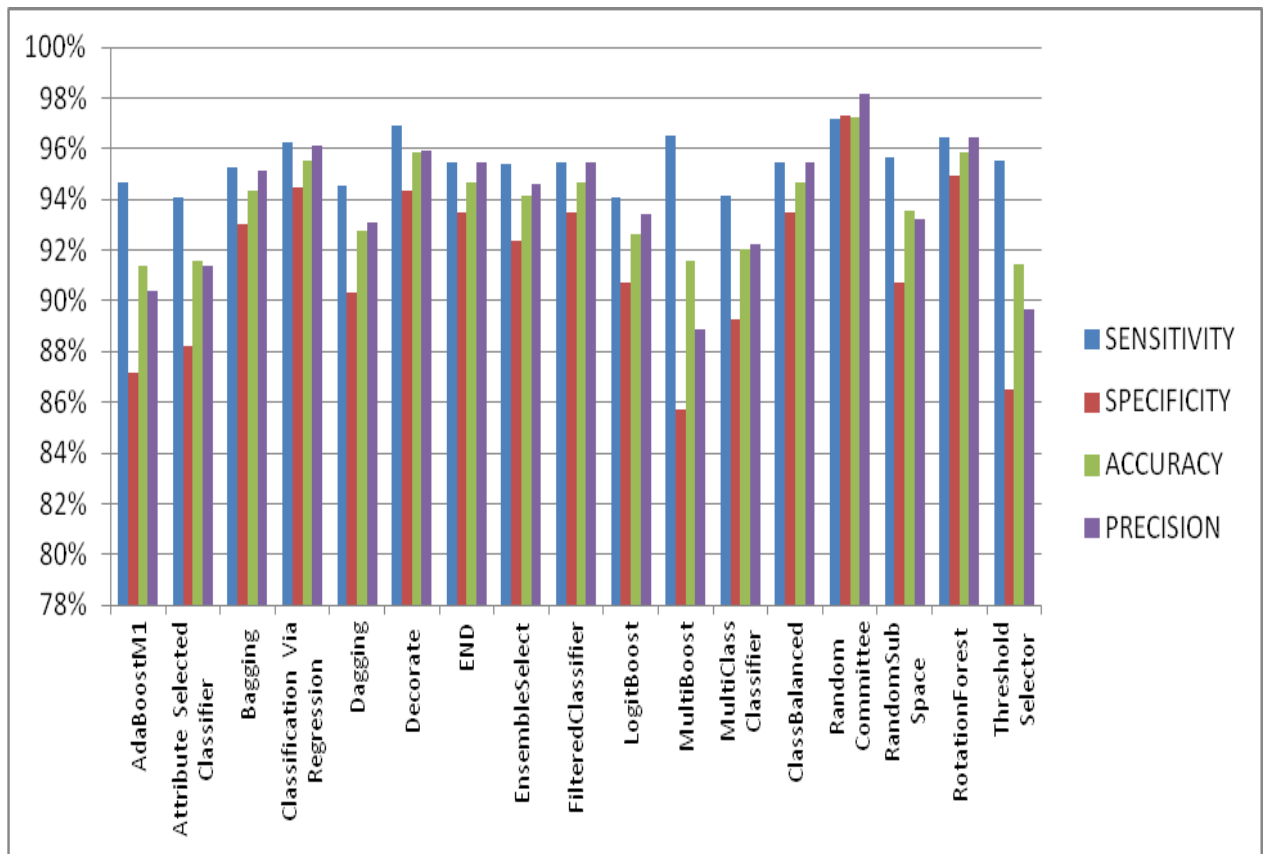

Figure 5. Comparison of various Meta classifiers 


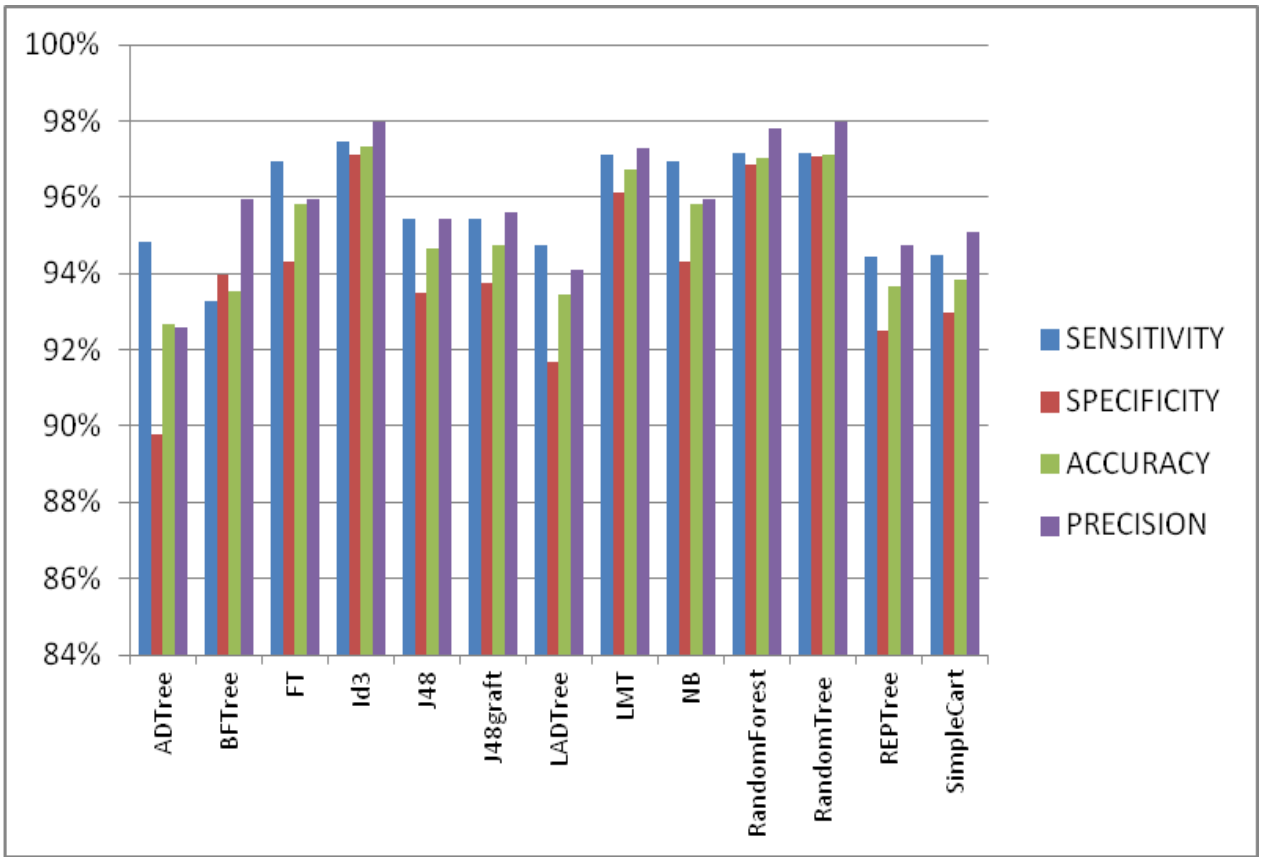

Figure 6. Comparison of various Tree classifiers

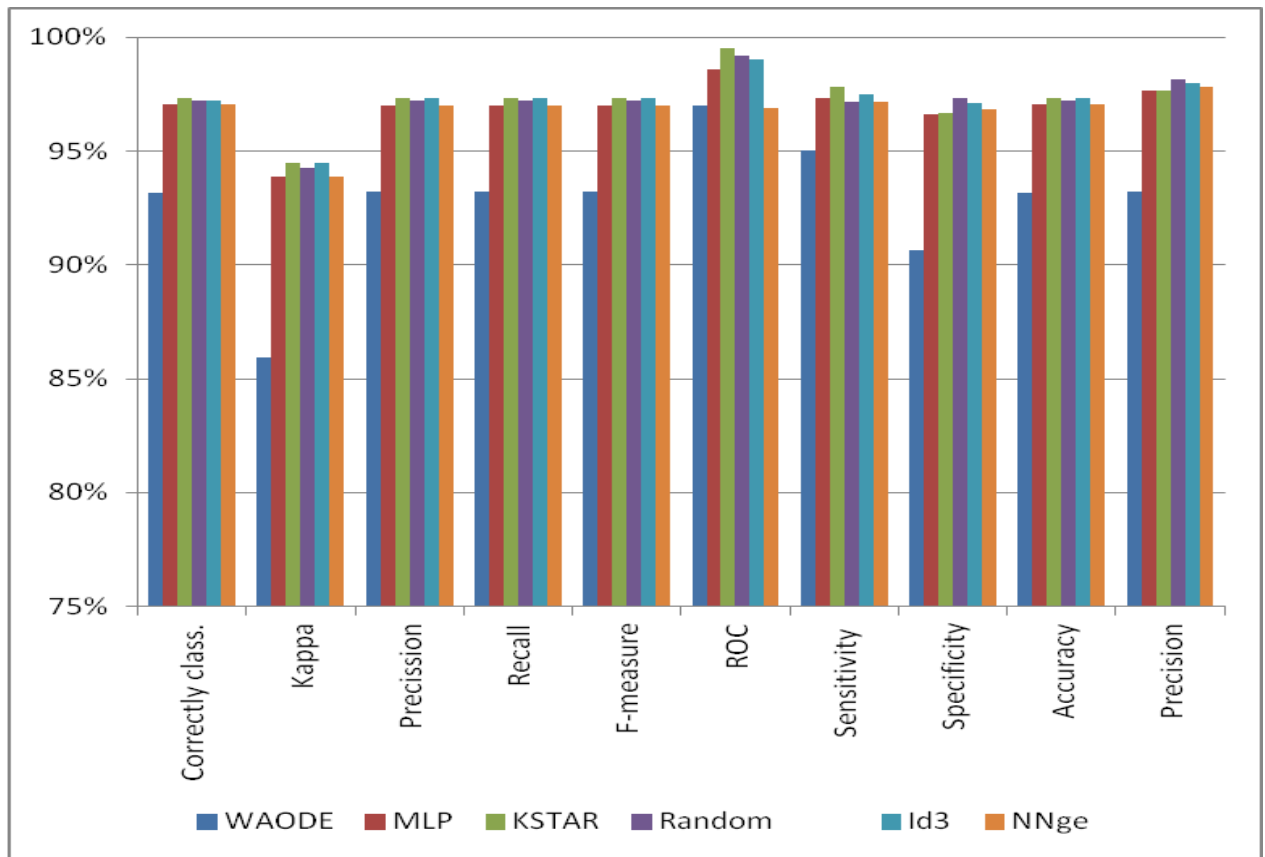

Figure 7. Comparison of various Rule classifiers 


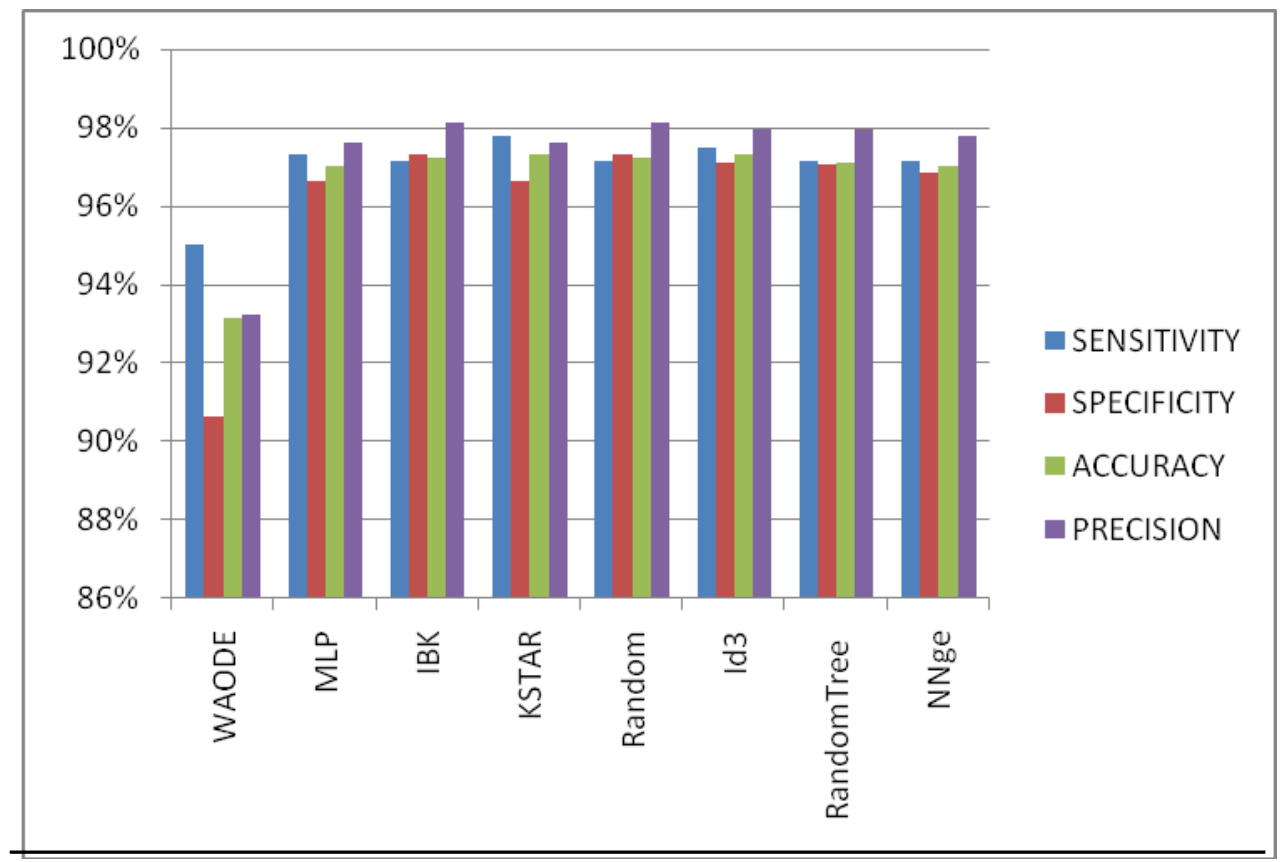

Figure 8. Comparison of Performance measures of best algorithmic models in 5 Classifiers

\section{RESULTS AND DISCUSSION}

A total of 59 models were used for classification of ischemic disease, and 16 models were found to be more efficient with accuracy when compared with the physician's diagnostic impression, gold standard and the kappa statistic (minimum of 0.736). The KSTAR algorithm showed the best diagnoses with the highest accuracy $97.32 \%$, sensitivity 0.98 , specificity 0.97 , kappa 0.95 and ROC 0.995 . The authors thus conclude that a CDSS can be developed to assist expert physicians in separating the positive and negative cases of heart disease. There was sensible agreement between the physician's diagnostic impression and the CDSS $\mathrm{k}=0.45(\mathrm{p}=0.0008)$. Sensitivity, specificity and accuracy are the commonly used statistical measures to illustrate the medical diagnostic test to enumerate how the test was good and consistent. Sensitivity evaluates the diagnostic test correctly at detecting a positive disease. Specificity measures how the proportion of patients without disease can be correctly ruled out. The association between both the sensitivity and specificity measures is defined by the graphical representation of the ROC curve, and this helps arrive at a decision to find the optimal model to determine the best threshold for the diagnostic test. Accuracy can be concluded with the aid of the sensitivity and specificity measures in the presence of prevalence.

Sensitivity $=\mathrm{TP} /(\mathrm{TP}+\mathrm{FN}) ;$ Specificity $=\mathrm{TN} /(\mathrm{TN}+\mathrm{FP})$; Accuracy $=(\mathrm{TN}+\mathrm{TP}) /(\mathrm{TN}+\mathrm{TP}+\mathrm{FN}+\mathrm{FP})$, where $\mathrm{TN}$ and $\mathrm{TP}$ are True Negatives and True Positives respectively. A comparison of 16 different best classifiers based on their performance measures; sensitivity, specificity, and accuracy are shown in Table 3.

Five AI techniques of classification (Bayes classifier, Function classifier, Meta classifier, Rule classifier, and Tree classifier) are used with a total of 59 models. The algorithms are run and parameters are obtained for further analysis. In the Bayes classifier, the WAODE classifier gives the best accuracy of 0.931. Table 1 illustrates the symptoms for heart disease identification. Figure 2 compares different algorithms of the
Bayes classifier on the basis of measures like sensitivity, specificity, accuracy, and precision. The WAODE algorithmic model is the best among the 7 algorithms in the Bayes Classifier with an accuracy of 93\%. Figure 3 shows a comparison between the Multilayer Perceptron (MLP), Radial Basis Network (RBF), Simple Logistic Classifier, Sequential Minimal Optimisation, and Voted Perceptron. MLP shows the best accuracy of $97 \%$. Figure 4 compares various measures between different lazy classifiers. KSTAR and IBK are equally better than the other three models with an accuracy of 97\%. Figure 5 gives an overview of the Meta Classifiers.

Figure 6 shows the performance measures of various tree classifying algorithms. The two algorithmic models that are equally better are ID3 and Randomtree, with an accuracy of 97\%. Figure 7 compares all models of the Rules Classifier. The best performer is the Nearest Neighbour like algorithm (NNge), with an accuracy of $97 \%$. Even though the precision of the ZeroR model is $100 \%$, its other performance measures are very low.

A comparison of the five best identified algorithms from each classifier type is displayed in Figure 8.

No studies investigating sudden deaths due to IHD without major tests like ECG, Stress tests, Cholesterol and Diabetes have been found in the literature. The literature includes various tests [23][24][25][26][27][28][29] for the identification of IHD. Through a systematic literature search, three incidence studies on $\mathrm{CHD}$ are identified within the Indian population [30]. The three incidence studies use different methods to attain data and so are not directly comparable. For a larger population, the need of the hour is to identify the IHD risk at the earliest and with a reduced number of feasible attributes. For this CDSS with attributes specially identified for the Indian population, diagnosis will be easier and quite manageable by experts, swiftly and efficiently. As variables for the measurement of social determinants of health depend on various factors like place of residence, race/ethnicity, gender, education, and socio- 
economic studies [31], further studies with different population groups are necessary.

\section{CONCLUSION}

The preliminary results of the work suggest that IHD can be diagnosed using the CDSS. KSTAR segregates the negative and positive cases more appropriately than the other methods. Sensitivity and accuracy are better than the other methods. It is essential to continue studying classifier accuracy, including attribute selection forIHD, in order to develop an electronic protocol along with CDSS in future. This study may help people to determine their heart disease risk, as it involves a simple procedure for decision making in an effective way by extracting hidden knowledge from a historical database. In India, various climatic, environmental and habitual conditions may also influence this case. Samples collected from one area may differ from others and can result in diverse sensitivity and accuracy. Future work will focus on collection of data sets from diverse locations and comparison of the results.

\section{ACKNOWLEDGEMENT}

The authors thank University of California, Irvine for providing benchmark data sets available as a free online resource.

\section{REFERENCES}

[1] V. Jomini, S. Oppliger-Pasquali, V. Wietlisbach, N. Rodondi, V. Jotterand, F. Paccaud, et al. "Contribution of major cardiovascular risk factors to familial premature coronary artery disease: the GENECARD project", J. Am. Coll. Cardiol., 2002, 40, 676-684.A. Asuncion, D. J. Newman, "UCI machine learning repository", 2007. Full text available here: http://www.ics.uci.edu/ mlearn/MLRepository.html (Accessed 16th July 2012).

[2] A. Asuncion, D. J. Newman, "UCI machine learning repository", 2007. Full text available here: http://www.ics.uci.edu/ mlearn/MLRepository.html (Accessed 16th July 2012).

[3] International Society of Cardiology and the Joint Subject Group on standardization of clinical naming in World Health Organization: Naming and diagnosis criteria of ischemic heart disease. Circulation, 1979, 59(3), 607.

[4] S. Vamadevan. A. Prabhakaran, D. Prabhakaran, "Coronary heart disease in Indians: Implications of the INTERHEART study", Indian. J. Med. Res., 2010, 132, 561-566.

[5] K. S. Reddy, B. Shah, C. Varghese, A. Ramadoss, "Responding to the threat of chronic diseases in India", Lancet, 2005, 366, 1744-9.

[6] R. Gupta, P. Joshi, V. Mohan, K. S. Reddy, S. Yusuf, "Epidemiology and causation of coronary heart disease and stroke in India", Heart, 2008, 94, 16-26.

[7] 7.S. Mendis, P. Puska, B. Norrving, "Global Atlas on Cardiovascular Disease Prevention and Control", World Health Organization, 2011, Full text available here: http://whqlibdoc.who.int/publications/2011/97892415643 73_eng.pdf (accessed 10th August 2012).

[8] E. A. Enas, "Clinical Implications: Dyslipidemia in the Asian Indian Population. Dyslipidemia in the Asian Indian Population: Unique aspects and implications for treatment", American Association of Physicians of Indian Origin, Oakbrook, 2002, Full text available here:
http://www.southasianheartcenter.org/docs/AAPImonogr aph.pdf (accessed 10th August 2012.

[9] S. O'Kelly, "Euroheart 2009", Heart Dis News, 2009, Full text available here: http://www.health.am/cardio/more/euroheart-2009/ (accessed 15th July 2012).

[10] European Society of Cardiology, "Obese adolescents have heart damage", Cardiology, 2012, Full text available here: http://medicalxpress.com/news/2012-05-obeseadolescents-heart.html (accessed 15th July 2012).

[11] J. R. Quinlan, "Induction of decision trees", Machine Learning, 1986, 1, 81-106.

[12] 12. J. R. Quinlan, "C4.5: Programs for Machine Learning", Morgan Kaufman, San Francisco, 1993.

[13] 13. V. S. Ajay, D. Prabhakaran, P. Jeemon, K. R. Thankappan, V. Mohan, L. Ramakrishnan, et al. "Prevalence and determinants of diabetes mellitus in the Indian Industrial population", Diabetic Med, 2008, 25, 1187-1194.

[14] 14. "India's Population 2012", 2012, Full text available here: http://www.indiaonlinepages.com/population/indiacurrent-population.html (accessed 26th July 2012).

[15] 15. Medical Council of India, Annual Report - 2009-10.

[16] 16. G. P. Liu, Y. Q. Wang, Y. Dong, et al., "Development and evaluation of Scale for heart system Inquiry of TCM" Chin. J. Integr. Med., 2009, 7(1), 1222-1225.

[17] E. García-Nietoa, A. L. Alba, B. E. Jourdan, B. Talbi, "Sensitivity- and specificity-based multiobjective approach for feature selection: Application to cancer diagnosis", Information Processing Letters, 2009, 109, 887-896.

[18] D. J. Newman, S. Hettich, C. L. Blake, C. J. Merz, "UCI repository of machine learning databases. Department of Information and Computer Science", University California Irvine, 1998.

[19] L. Breiman, J. Friedman, R. Olshen, C. Stone, "Classification of Regression Trees", Wadsworth, 1984.

[20] M. Hall, E. Frank, G. Holmes, B. Pfahringer, P. Reutemann, I. H. Witten, "The WEKA data mining software: an update", SIGKDD Explorations, 2009, 11(1), 10-18.

[21] I. H. Witten, E. Frank, "Data Mining: Practical Machine Learning Tools and Techniques", 2nd ed., Morgan Kaufmann, San Francisco, 2005.

[22] E. Massad, "A teoria bayesiana no diagnóstico medico", in: "Métodos quantitativos em medicina" (Ed. E. Massad, R. X. Menezes, P. S. P. Silveira, N. R. S. Ortega), Barueri (SP), Manole, 2004, 189-205.

[23] P. K. Anooj, "Clinical decision support system: Risk level prediction of heart disease using weighted fuzzy rules", J. King. Saud. Univ., - Computer and Information Sciences 2012, 24, 27-40.

[24] European ST-T Database Directory. Pisa, Italy: S.T.A.R., 1991.

[25] M. G. Tsipouras, C. Voglis, D. I. Fotiadis, "A Framework for Fuzzy Expert System Creation-Application to 
Cardiovascular Diseases", IEEE Transactions on BioMedical Engineering, 2007, 54, 11.

[26] A. Gharaviri, M. Teshnehlab, H. A. Moghaddam, "Ischemia Detection via ECG Using ANFIS", Conf Proc. IEEE. Eng. Med. Biol. Soc., 2008, 1163-1168.

[27] M. A. Karaolis, J. A. Moutiris, D. Hadjipanayi, C. S. Pattichis, "Assessment of the Risk Factors of Coronary Heart Events Based on Data Mining With Decision Trees", IEEE Transactions on Information Technology in BioMedicine, 2010, 14, 3.

[28] D. Frenkel, J. Nadal, "Ischemic episode detection using an artificial neural network trained with isolated ST-T segments", Comp. Cardiol., 1999, 53-56.

[29] M. Piao, H. G. Lee, G. Y. Sohn, G. Pok, K. H. Ryo, "Emerging Patterns based Methodology for Prediction of
Patients with Myocardial Ischemia", Sixth International Conference on Fuzzy Systems and Knowledge Discovery, 2009, 1, 174-178.

[30] National Cardiovascular Disease Database (Sticker No: SE / 04 / 233208), Supported by Ministry of Health \& Family Welfare, Government of India and World Health Organization, 2011, Full text available here: http://www.whoindia.org/ (accessed 10th August 2012).

[31] P. Jeemon, K. S. Reddy, "Social determinants of cardiovascular disease outcomes in Indians", Indian. J. Med. Res,. 2010, 132, 617-622.

[32] () 2011 by Maejo University, San Sai, Chiang Mai, 50290 Thailand. Reproduction is permitted for noncommercial purposes. 